\title{
Characteristics of the dust trail of 67P/Churyumov-Gerasimenko: an application of the IMEX model ${ }^{\star}$
}

\author{
R. H. Soja ${ }^{1}$, M. Sommer ${ }^{1}$, J. Herzog ${ }^{1}$, J. Agarwal ${ }^{2}$, J. Rodmann ${ }^{3}$, R. Srama ${ }^{1}$, J. Vaubaillon ${ }^{4}$, P. Strub ${ }^{1}$, A. Hornig ${ }^{1,5}$, \\ L. Bausch ${ }^{5}$, and E. Grün $6,7,8$ \\ ${ }^{1}$ Institute of Space Systems, University of Stuttgart, Pfaffenwaldring 29, 70569 Stuttgart, Germany \\ e-mail: soja@irs.uni-stuttgart.de \\ 2 Max-Planck-Institut für Sonnensystemforschung, Justus-von-Liebig-Weg 3, 37077 Göttingen, Germany \\ 3 University of Göttingen, Institute for Astrophysics, 37077 Göttingen, Germany \\ 4 IMCEE, 77 avenue Denfert Rochereau, 75014 Paris, France \\ 5 Aerospace Research, Linder Höhne, 51147 Köln, Germany \\ 6 Max-Planck-Institut für Kernphysik, Saupfercheckweg 1, 69117 Heidelberg, Germany \\ 7 Laboratory for Atmospheric and Space Physics, University of Colorado, Boulder, CO 80303, USA \\ 8 Institute for Modeling Plasmas, Atmospheres, and Cosmic Dust (IMPACT), University of Colorado, Boulder, CO 80303, USA
}

Received 25 March 2015 / Accepted 23 May 2015

\begin{abstract}
Context. Here we describe a new model of the dust streams of comet 67P/Churyumov-Gerasimenko that has been developed using the Interplanetary Meteoroid Environment for Exploration (IMEX). This is a new universal model for recently created cometary meteoroid streams in the inner solar system.

Aims. The model can be used to investigate characteristics of cometary trails: here we describe the model and apply it to the trail of comet 67P/Churyumov-Gerasimenko to develop our understanding of the trail and assess the reliability of the model.

Methods. Our IMEX model provides trajectories for a large number of dust particles released from $\sim 400$ short-period comets. We use this to generate optical depth profiles of the dust trail of comet 67P/Churyumov-Gerasimenko and compare these to Spitzer observations of the trail of this comet from 2004 and 2006.

Results. We find that our model can match the observed trails if we use very low ejection velocities, a differential size distribution index of $\alpha \approx-3.7$, and a dust production rate of 300-500 $\mathrm{kg} \mathrm{s}^{-1}$ at perihelion. The trail is dominated by mm-sized particles and can contain a large proportion of dust produced before the most recent apparition. We demonstrate the strength of IMEX in providing time-resolved histories of meteoroid streams. We find that the passage of Mars through the stream in 2062 creates visible gaps. This indicates the utility of this model in providing insight into the dynamical evolution of streams and trails, as well as impact hazard assessment for spacecraft on interplanetary missions.
\end{abstract}

Key words. comets: individual: 67P/Churyumov-Gerasimenko - meteorites, meteors, meteoroids - comets: general

\section{Introduction}

Dust trails in the vicinity of comets were first observed by the Infrared Astronomical Satellite (IRAS; Sykes et al. 1986). Subsequent infrared observations found evidence for dust trails associated with $>80 \%$ of Jupiter-family comets (Reach et al. 2007). These trails form fine-structure on top of the infrared emission of the interplanetary background cloud. They consist of the largest cometary particles (with sizes of $\sim 0.1 \mathrm{~mm}-\mathrm{cm}$ ), which are ejected at low speeds and remain very near the comet orbit for several revolutions: they are distinct from the particles in the comet tail, which are much smaller and disperse rapidly as a result of higher ejection speeds and radiation pressure.

Kresak (1993) clarified the link between trails and meteor storms, showing that the width of debris trails matched the duration of meteor storms (less than an hour) and not meteor

\footnotetext{
* A movie is available in electronic form at http://www. aanda.org
}

showers (days), and that the spatial density of trail particles better matches the high rates of meteors seen during meteor storms.

The trail of 67P/Churyumov-Gerasimenko is of particular interest at present, as a result of ESA's Rosetta mission, first results from which are already available concering the comet's dust production (Rotundi et al. 2015; Schulz et al. 2015). There exists a large body of research on modeling the dust trail (Agarwal et al. 2007, 2010; Ishiguro 2008; Kelley et al. 2008; Fulle et al. 2010).

The project Interplanetary Meteoroid Environment for Exploration (IMEX) "Dust Streams in Space" aims to extend ESA's Interplanetary Meteoroid Environment Model (IMEM; which describes the interplanetary background dust cloud, Dikarev et al. 2005) by characterizing these trails. This model is ideal for studying cometary dust trails, as observed in infrared images. Here we compare the results of the IMEX model to Spitzer observations of the trail of comet $67 \mathrm{P} /$ ChuryumovGerasimenko, to define charateristics of the trail and to demonstrate the potential of this model. 
The paper therefore first describes the IMEX model (Sect. 2) before demonstrating its application to the trail of 67P/Churyumov-Gerasimenko (Sect. 3) and finally discussing the implications for the trail of this comet (Sect. 4) and future applications for the IMEX model (Sect. 5).

\section{The IMEX model}

Here we describe IMEX, which provides a model for the dust trails of 420 short-period comets. We include details of the input cometary population, dust emission process, the integration and storing of dust trajectories, and the use of the model.

\subsection{Cometary orbits}

We have generated streams for 420 Jupiter-family, Halley-type, and Encke-type comets in the JPL Small Body Database (SBDB) as of 1 August 2013, which have perihelion distances $q<3 \mathrm{AU}$, semimajor axes $a<30$ AU and defined total visual magnitudes. Comets that do not fit these parameters either do not come close enough to the Sun to generate dust under our assumption of water-generated dust production inward of $3 \mathrm{AU}$, fall outside our region of interest, or do not contain the magnitude information we require to calculate the dust production of the comet. We removed a very small number of comets with highly unpredictable orbital behavior, such as exceeding an eccentricity of 1 for a long period of their orbit, which in many cases would limit the ability of the comet to form a trail that would return to the inner solar system. The final data sets include 362 Jupiter-family comets, 40 Halley-type comets, and 18 Encke-type comets.

Particles are emitted from Halley-type comets starting in 1700, and for Jupiter-family and Encke-type comets starting in 1850. These limits reflect that we expect the most recent dust to be most important, as well as the maximum size of the database that we can support. Dust emission in the model ends in 2080. In a few cases, these limits are modified to account for significant changes in the comet orbits, which can throw the comets into or out of the inner solar system. The limits are additionally modified to introduce fragments after their expected creation dates.

Orbital data for comets are not available in SPK format (as accessible by NASA NAIF SPICE) from JPL HORIZONS outside the years 1900-2100. This reflects the level of uncertainty that exists beyond this time period. For a small number $(\sim 20)$ of major comets we use JPL HORIZONS orbits that are constructed by piecing together different comet "solutions" given by the HORIZONS database. For all other comets the orbits have been computed using the MODUST code (Rodmann 2006). The gravitational potential of the Sun and planetary perturbations are included as the most important force terms. Non-gravitational forces on the comet nuclei (Yeomans et al. 2004) are not well known for most comets and therefore are not included. MODUST uses a Hermite scheme with individual timesteps for integrating the comets' motion. A detailed description of the method and numerical approach is given in Ertel et al. (2012).

\subsection{Emission of dust}

Particles are emitted for each apparition of each comet within the time frames specified above. They are randomly emitted from the sunlit hemisphere of each comet, at 251 locations that are evenly spaced in true anomaly within $3 \mathrm{AU}$ of the
Sun. Hundreds of thousands of particles are ejected for each comet: 28 000 per comet apparition for Halley-type comets and $\sim 14000$ for other comets. Twice as many particles are emitted at each emission point for Halley-type comets because we integrate many particles from these comets for longer durations, which increases the dispersion. The random particle emission uses a sphere point-picking method to ensure there is no bunching of particles emitted near the poles.

Particle ejection velocities are computed using the velocity model from the hydrodynamic comet emission model of Crifo \& Rodionov (1997), given by the following set of equations:

$$
\begin{aligned}
V & =W(T) \times \Phi\left(a_{\mathrm{d}}, a_{*}\right) \\
W & =\sqrt{\frac{\gamma+1}{\gamma-1} \frac{\gamma K_{\mathrm{B}} T}{m_{\mathrm{g}}}} \\
\Phi & \simeq \frac{1}{1.2+0.72 \sqrt{a_{\mathrm{d}}, a_{*}}} \\
a_{*} & =\frac{m_{\mathrm{g}}(1-A) c_{\odot}}{\rho \alpha_{\mathrm{s}} L_{\mathrm{s}} V_{\mathrm{g}}^{0}} R_{\mathrm{c}} \frac{f\left(r_{\mathrm{h}}\right) \cos z}{r_{\mathrm{h}}^{2}}
\end{aligned}
$$

where $\gamma=\frac{4}{3}$ is the specific heat ratio that characterizes $\mathrm{H}_{2} \mathrm{O}$ molecules, $K_{\mathrm{B}}$ is the Boltzmann constant, $m_{\mathrm{g}}$ is the mass of a gas molecule (assumed to be $\mathrm{H}_{2} \mathrm{O}$ ), $A$ is the comet albedo (we take $5 \%$ ), $c_{\odot}$ is the solar constant, $\alpha_{\mathrm{S}}$ is the coefficient of sublimation (assumed to be 1), $L_{\mathrm{S}}$ is the latent heat of sublimation for $\mathrm{H}_{2} \mathrm{O}, V_{\mathrm{g}}^{0} \simeq \sqrt{\gamma K_{\mathrm{B}} T / m_{\mathrm{g}}}$ is the initial gas velocity, $T$ is the temperature, $z$ is the solar zenith angle, $a_{\mathrm{d}}$ is the dust radius, $\rho$ is the particle bulk density, $r_{\mathrm{h}}$ is the heliocentric distance (AU), and $R_{\mathrm{c}}$ is the comet radius.

Our assumption is that cometary dust emission is driven by water gas production. We therefore do not consider cometary dust emitted as a result of the sublimation of other gases outside of $3 \mathrm{AU}$, or as a result of comet fragmentation. In many cases, including 67P/Churyumov-Gerasimenko (Rotundi et al. 2015; Schulz et al. 2015), comets can emit dust farther away from the Sun. Gundlach et al. (2015) found that $\mathrm{H}_{2} \mathrm{O}$ can only contribute to dust production for this comet out to $2.5 \mathrm{AU}$ and that thereafter $\mathrm{CO}$ and $\mathrm{CO}_{2}$ are responsible. The dust production rate is expected to be significantly lower in this outer region, and therefore this emission is expected to provide minimal contributions to the resulting meteoroid streams.

Particles have eight different masses according to a logarithmic distribution, such that there are more mass intervals at lower masses, where the variations between particles of different masses are greatest: $1 \times 10^{-8}, 1.64 \times 10^{-8}, 4.39 \times 10^{-8}$, $1.93 \times 10^{-7}, 1.39 \times 10^{-6}, 1.64 \times 10^{-5}, 3.16 \times 10^{-4} \mathrm{~kg}$, and $1 \times 10^{-2} \mathrm{~kg}$. Thus, particle radii fall between $100 \mu \mathrm{m}$ and $1 \mathrm{~cm}$. We assume a bulk density of $\rho=1000 \mathrm{~kg} \mathrm{~m}^{-3}$ and a comet nucleus density of $500 \mathrm{~kg} \mathrm{~m}^{-3}$. For comets without a known radius, we assume a value of $1 \mathrm{~km}$.

The same number of test particles are emitted for each location along the orbit of each comet. We determine how many "real" particles each test particle represents using the dust output for the comet, a mass distribution, and the emission direction of the particles relative to the solar zenith direction. We define the "multiplication factor" to be the number of particles that a given test particle represents. This is calculated in three steps:

1. calculating the total amount of dust emitted in the true anomaly region defined around a given emission point (Sect. 2.2.1); 
2. using a mass distribution to determine how much dust is emitted in each mass range (Sect. 2.2.2), using the total mass emitted at this true anomaly;

3. splitting this between each test particle by multiplying by a scaling factor that accounts for the distribution of dust emission relative to the angle from the subsolar point.

We describe the calculation of the first two factors in the following sections. The final factor is calculated assuming that the number of emitted particles is proportional to the cosine of the latitude of the emitted particles from the subsolar point.

\subsubsection{Dust production rate}

We estimate the water production rate using the total magnitude and use a gas-to-dust ratio to convert this to a dust production rate. We use this method because the $[A(\theta) f \rho]$ parameter, which provides a method for estimating dust production information for a comet by correcting for various observational parameters (A'Hearn et al. 1984), is only known for a small number of comets. We use the empirical formula for the correlation between visual magnitudes reduced to a geocentric distance of $1 \mathrm{AU} m_{\mathrm{H}}$ and water production rates $Q_{\left[\mathrm{H}_{2} \mathrm{O}\right]}$ given by Jorda et al. (2008):

$\log Q_{\left[\mathrm{H}_{2} \mathrm{O}\right]}=30.675(0.007)-0.2453(0.0013) m_{\mathrm{H}}$

where $m_{\mathrm{H}}$ is calculated from the JPL Small Body Database using both the total magnitude $M_{1}$ and the magnitude heliocentric distance slope parameter $K_{1}$.

As a representative value, at the perihelion (1.29 AU) of the 2002.6 apparition of Comet 67P/Churyumov-Gerasimenko we find $Q_{\left[\mathrm{H}_{2} \mathrm{O}\right]}=3.7 \times 10^{27} \mathrm{~mol} \mathrm{~s}^{-1}$. This is slightly lower than reported observations for various apparitions, which range from $6 \times 10^{27} \mathrm{~mol} \mathrm{~s}^{-1}$ to $2 \times 10^{28} \mathrm{~mol} \mathrm{~s}^{-1}$ (Crovisier et al. 2002; Feldman et al. 2004; Mäkinen 2004; Schleicher 2006), with values dependent on the heliocentric distance of the observation, but all within 1.24-1.31 AU.

Dust-to-gas mass ratios can be estimated for individual comets. Various publications provide values for a range of comets (Singh et al. 1992; Sanzovo et al. 1996, 2010; A'Hearn et al. 1995; Langland-Shula \& Smith 2011). However, these publications only give numbers for a small range of comets. Dust-to-gas mass ratios mostly range from $0.1-3$, although higher values are possible (Rotundi et al. 2015 found $4 \pm 2$ for $67 \mathrm{P} /$ Churyumov-Gerasimenko at heliocentric distances between 3.7 and 3.4 AU). A'Hearn et al. (1995) showed that these ratios appear to be strongly dependent on heliocentric distance. Given the large uncertainties in cometary dust-to-gas ratios, we use a value of 1 . Deviations from this can be considered in the analysis of individual comet streams.

The dust production for each of the 251 emission points in true anomaly $v$ is represented by the dust production in the range $v-\frac{\Delta v}{2}$ to $v+\frac{\Delta v}{2}$, where $\Delta v$ is the step size between true anomaly values (Fig. 1). We find a maximum dust production at perihelion of $\sim 100 \mathrm{~kg} \mathrm{~s}^{-1}$ for $67 \mathrm{P} /$ Churyumov-Gerasimenko with dust to gas ratio 1 . Values derived from observations vary widely: Kidger (2004) found $\sim 50 \mathrm{~kg} \mathrm{~s}^{-1}$ at perihelion for the 2002 apparition, Ishiguro (2008) found $\sim 180 \mathrm{~kg} \mathrm{~s}^{-1}$, Fulle et al. (2010) found $\sim 500 \mathrm{~kg} \mathrm{~s}^{-1}$, while Agarwal et al. (2010) found a value of $\sim 1000 \mathrm{~kg} \mathrm{~s}^{-1}$ at perihelion. (a)
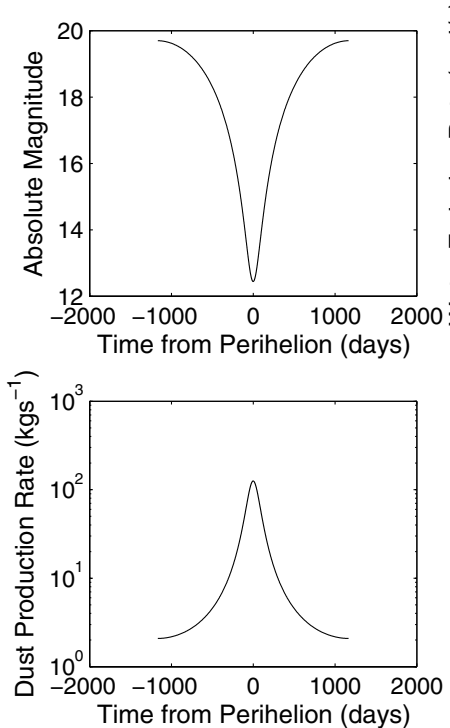

(c) (b)
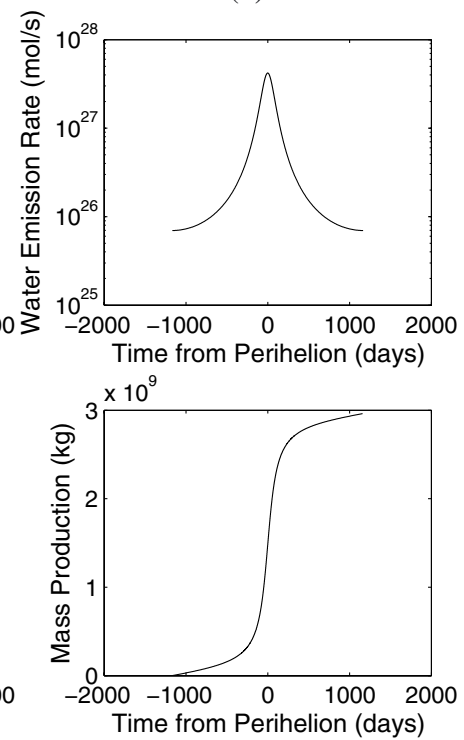

(d)

Fig. 1. Model dust production for the 1963 apparition of Churyumov-Gerasimenko. Figures display the change of a) the total magnitude; b) the water production rate; c) the dust production rate; and d) the cumulative dust production rate as a function of time from perihelion.

\subsubsection{Mass distribution}

We use the mass distribution model of Divine \& Newburn (1987), as applied for the purpose of modeling the trail of comet 67P/Churyumov-Gerasimenko by Agarwal et al. (2007, 2010). This is a cumulative mass distribution:

$F(m)=\left(\frac{(1+x)^{b-1}}{x^{b}}\right)^{a c}$

with

$x=\left(\frac{m}{m_{\mathrm{t}}}\right)^{1 / c}$.

Following Agarwal et al. (2007), we choose $m_{\mathrm{t}}=10^{-13} \mathrm{~kg}$ and $c=2$, and use $a$ and $b$ such that the index of the cumulative mass distribution is $\gamma=a b=0.26$ for light particles, and $\gamma=a$ for heavy particles. For heavy particles we use an index for the differential size distribution $\alpha=-3.7$, where $\alpha=-3 \gamma-1$. The constants are therefore $a=\gamma, b=0.26 / a, c=2$ and $m_{\mathrm{t}}=10^{-13} \mathrm{~kg}$. To constrain the particles that contribute to the total dust mass production, we use a minimum particle mass of $10^{-20} \mathrm{~kg}$. This distribution allows us to calculate the number of particles emitted in each mass (Fig. 2).

These calculations require a maximum radius of particles that can be emitted from the comet. This can be estimated by equating the gas drag and gravitational forces on an ejected particle at the comet surface. We use the formula given by Molina (2010) for a cosine emission distribution.

\subsection{Particle integration: creation of a dataset of meteoroid streams}

The emitted particles are individually integrated from their creation time up until 2080. Particle integrations are performed using a Runge-Kutta-Nyström 7(6) integrator with variable step size (Dormand \& Prince 1978). The emitted 


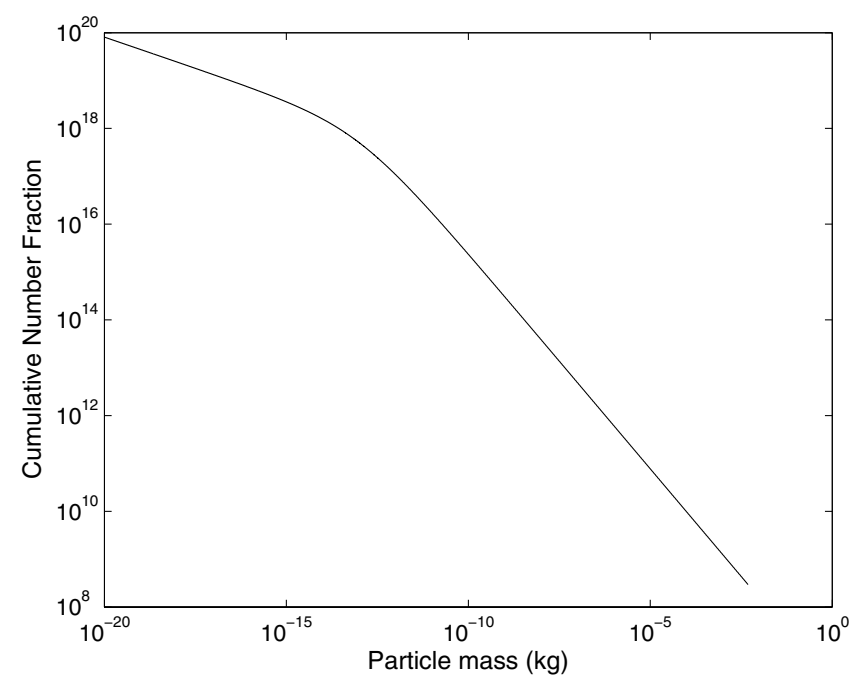

Fig. 2. Cumulative number distribution.

particles are individually integrated including gravity of the Sun and eight planets, as well as radiation pressure and the Poynting-Robertson effect (including a factor for solar wind drag of 0.3 Gustafson 1994). Relativistic corrections to solar gravity are included. Planet positions are taken from JPL's Development Ephemeris 430. The integrator is designed to record the particle states at a number of "save points" per orbit (more near perihelion and near close planetary encounters). This creates a database from which the full trajectories of each particle from each comet can be reconstructed between 1980 and 2080. The particles are integrated at earlier times, but are not saved to limit the size of the database. The database consists of a set of binary files for each comet, containing position and velocity information for integrated particles.

The calculations are completed using the Constellation distributed computing platform, in which the computational load is shared over many individual computers. The work is split into packages of particles called work units, which are distributed among participating computers. Constellation ${ }^{1}$ is a system in which public users can donate their idle time on their private computers to provide distributed computing capability to aerospace-related science and engineering projects. Distribution and processing of the work units is managed by the Berkeley Open Infrastructure for Network Computing (BOINC) system developed at the University of Berkeley. It was originally designed to enable distributed computing for the SETI at home project. At present, a wide variety of scientific projects use the BOINC platform. Currently, Constellation has 13000 volunteers who donate time on more than 66000 computers, thus providing about 5 TeraFLOPs.

\subsection{Application of IMEX}

The model provides a database of integrated trajectories for thousands of dust particles that are emitted during each apparition of 420 short-period comets. The dust trajectories can be retrieved from the database on a given date between 1980 and 2080, either for all particles from one comet, or for all particles near a position in the inner solar system. These can be used to study comet trails and meteor showers and to analyze the streams that impact a given location at a particular time.

\footnotetext{
1 http://aerospaceresearch.net/constellation/
}

Additionally, when updated observational data are available for an individual comet, the original structure of IMEX can be used to recalculate the stream to provide a more accurate database entry. New comets can also be added to the model in this way.

We now describe the application of the model to the trail of comet $67 \mathrm{P} /$ Churyumov-Gerasimenko. This requires using the database to extract the stream of this comet on the dates of Spitzer images of the trail and comparing these observations with the results from IMEX. We adjust various parameters of the comet emission process to best match the observations. We use this case to discuss the accuracy and limitations of IMEX.

\section{Comparison between the model and observations of the trail of comet 67P/Churyumov-Gerasimenko}

Here we compare the results of our model to Spitzer $24 \mu \mathrm{m}$ observations of the trail of comet 67P/Churyumov-Gerasimenko by Kelley et al. (2008; observation taken on 4 February 2004) and Agarwal et al. (2010; 29 August 2005 and 9 April 2006). The infrared images themselves can be found in Fig. 2 of Kelley et al. (2008) and Figs. 2 and 3 of Agarwal et al. (2010). Particles were emitted from 67P/Churyumov-Gerasimenko and their orbits were integrated under gravitational and radiation forces to determine their final positions on these dates. Before 1959, the comet's orbit remained largely outside of 3 AU. A close encounter with Jupiter in this year shifted the comet into its current orbit. Particles were therefore only emitted after 1959. The orbit was taken from JPL Horizons. The JPL Small Body Database provides a visual total magnitude of 11.4 and a magnitude slope parameter of 11 , which we used to define the dust production rate. We tested three differential size distribution indices $\alpha$ and three velocity models. Two of the differential size distribution indices, $\alpha=-3.5$ and $\alpha=-4.1$, are the values found respectively by Kelley et al. (2008) and Agarwal et al. (2010) to best match their data sets. The third, $\alpha=-3.7$, is an intermediate value. The first velocity model is that of Crifo \& Rodionov (1997), which is used for the main IMEX model. We additionally used velocity models derived using these Spitzer observations. They provide ejection velocities that are much lower than those obtained from the model of Crifo \& Rodionov (1997). This is in line with early measurements by Rosetta (Rotundi et al. 2015). The velocity model of Kelley et al. (2008) is

$V_{\mathrm{ej}}=500 \sqrt{\beta / r_{\mathrm{h}}} \cos z \mathrm{~ms}^{-1}$,

and the velocity model of Agarwal et al. (2010)

$V_{\mathrm{ej}}=500 \sqrt{\beta} r_{\mathrm{h}}^{-3} \mathrm{~ms}^{-1}$,

where $\beta$ is the ratio between radiation and gravitational forces and $z$ is the solar zenith angle of particle emission.

We converted the resulting stream into a trail on the sky (Fig. 3) and calculated the optical depth using (1) the number of test particles in each area of sky; and (2) the surface area of these test particles. The surface area contribution was calculated using the mass distributions described in Sect. 2.2.2 and additionally includes a particle weighting that accounts for the variation in the number of particles with angle from the subsolar point. We used an absorption efficiency $Q=1$, which is valid here because our particles have $\frac{2 \pi a}{\lambda} \geqslant 1$, where $a$ is the particle radius and $\lambda$ is the wavelength (Vaubaillon \& Reach 2010).

We binned particles in each unit of sky, adding their weighted surface areas. The bin size was determined by the pixel 
Table 1. Calculated maximum trail optical depths and ratios between observed and calculated trail optical depths for three velocity models (Crifo \& Rodionov 1997; Kelley et al. 2008; Agarwal et al. 2010) and two size distribution indices.

\begin{tabular}{llcccccc}
\hline \hline Year & Velocity model & \multicolumn{3}{c}{ Optical depth $\tau$} & \multicolumn{3}{c}{ Ratio between observed and modeled $\tau$} \\
Size distn & & $\alpha=-3.5$ & $\alpha=-3.7$ & $\alpha=-4.1$ & $\alpha=-3.5$ & $\alpha=-3.7$ & $\alpha=-4.1$ \\
\hline \multirow{2}{2}{2004} & Crifo & $2.5 \times 10^{-9}$ & $2.1 \times 10^{-9}$ & $1.2 \times 10^{-10}$ & 19 & 22 & 40 \\
& Kelley & $5.3 \times 10^{-9}$ & $4.4 \times 10^{-9}$ & $1.9 \times 10^{-9}$ & 8.8 & 11 & 24 \\
& Agarwal & $1.3 \times 10^{-9}$ & $1.0 \times 10^{-9}$ & $5.0 \times 10^{-9}$ & 3.5 & 4.4 & 9.1 \\
2005 & Crifo & $1.4 \times 10^{-9}$ & $3.7 \times 10^{-10}$ & $7.1 \times 10^{-10}$ & 11 & 13 & 25 \\
& Kelley & $3.2 \times 10^{-9}$ & $2.4 \times 10^{-9}$ & $4.6 \times 10^{-10}$ & 4.7 & 6.4 & 16 \\
& Agarwal & $6.8 \times 10^{-9}$ & $5.0 \times 10^{-9}$ & $9.7 \times 10^{-9}$ & 2.2 & 3.0 & 7.6 \\
2006 & Crifo & $6.5 \times 10^{-10}$ & $5.7 \times 10^{-10}$ & $3.4 \times 10^{-10}$ & 17 & 19 & 33 \\
& Kelley & $2.0 \times 10^{-9}$ & $1.7 \times 10^{-9}$ & $7.9 \times 10^{-10}$ & 5.4 & 6.6 & 14 \\
& Agarwal & $2.6 \times 10^{-9}$ & $2.1 \times 10^{-9}$ & $1.0 \times 10^{-9}$ & 4.2 & 5.2 & 11 \\
\hline
\end{tabular}

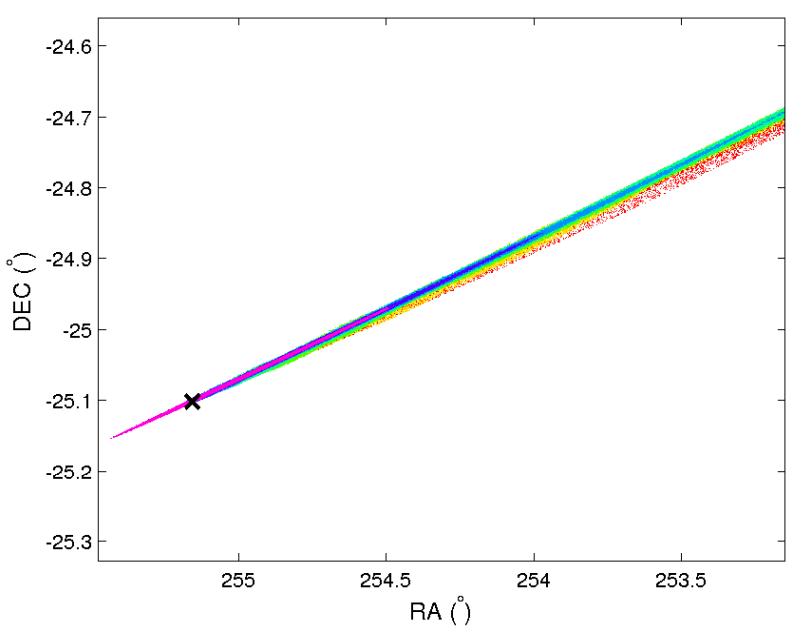

Fig. 3. RA and Dec of test particles for the trail of 67P/ChuryumovGerasimenko on 9 April 2006. Each color represents a different mass bin: the smallest grains are red; the largest are magenta.

size and binning of the data. For the observation in 2004 reported by Kelley et al. (2008), this binning is $21^{\prime \prime} \times 7.4^{\prime \prime}$. For the observations in 2005 and 2006 described by Agarwal et al. (2010), the binning is $7.4^{\prime \prime} \times 7.4^{\prime \prime}$ and $272^{\prime \prime} \times 4.9^{\prime \prime}$, respectively. The observational data for 2006 were rebinned to match the statistics of our model profile. The 2004 and 2005 observations require very small bins to resolve the peak. The 2004 and 2006 profiles are relatively insensitive to the binning size. The 2005 data are more sensitive to the selected binning, and consequently, we place less confidence in these results. We compared our modeled trail to the observations, considering both (1) the maximum optical depth (or brightness); and (2) trail profiles along and perpendicular to the projected comet orbit.

\subsection{Maximum optical depth}

We compared the maximum optical depths given by the IMEX model to the values derived from the Spitzer observations (Table 1) for models using three velocity models and three size distribution indices $\alpha$. The Spitzer observations provide the maximum surface brightness of the trail in MJy/sr. We used Planck's law to convert these to optical depths (Table 2). The temperature as a function of heliocentric distance was calculated from the equilibrium between absorbed solar and emitted thermal radiation, assuming that the particles are characterized by a Bond albedo of 0.18 , which was derived from a geometric albedo of 0.04 and the phase function given by Divine (1981).
Table 2. Observational circumstances, calculated equilibrium temperatures, and derived optical depths of comet 67P/ChruyumovGerasimenko.

\begin{tabular}{lccc}
\hline \hline Date & $r(\mathrm{AU})$ & $T(\mathrm{~K})$ & $\tau$ \\
\hline 4 February 2004 & 4.47 & 125.5 & $4.6 \times 10^{-8}$ \\
29 August 2005 & 5.69 & 111.2 & $1.5 \times 10^{-8}$ \\
9 April 2006 & 5.66 & 111.5 & $1.1 \times 10^{-8}$ \\
\hline
\end{tabular}

In Table 1 we provide the ratios between the observed and modeled optical depths. The velocity model of Crifo \& Rodionov (1997) gives optical depths that are factors of 13-40 lower than observed. The velocity model of Agarwal et al. (2010) produces optical depths that are lower than observed by factors $2.2-4.2$ for $\alpha=-3.5,3.0-5.2$ for $\alpha=-3.7$, and $8-11$ for $\alpha=-4.1$.

In all cases, the modeled optical depths are lower than those observed. This may be accounted for by our choice of a dust-togas mass ratio of 1 . Various publications suggest that this comet has a high dust-to-gas mass ratio (Sykes \& Walker 1992; Weiler et al. 2004; Snodgrass et al. 2013), perhaps 3 or 4. More recently, new Rosetta data suggest a dust-to-gas ratio of $4 \pm 2$ at heliocentric distances between 3.7 and 3.4 AU (Rotundi et al. 2015), although this may change as the comet moves toward perihelion. We can also assess this by direct comparison of the dust production rates. In Sect. 2.2.1 we discussed that our dust production rate at perihelion is about $100 \mathrm{~kg} \mathrm{~s}^{-1}$. This section contains other observational results for the water gas and dust production rates that confirm that our model produces weaker activity for this comet than is observed. Our comparison between the IMEX model and the Spitzer data therefore implies a dust production rate for comet $67 \mathrm{P} /$ Churyumov-Gerasimenko of $200-400 \mathrm{~kg} \mathrm{~s}^{-1}$, $300-500 \mathrm{~kg} \mathrm{~s}^{-1}$, or $800-1100 \mathrm{~kg} \mathrm{~s}^{-1}$, depending on $\alpha$. These are in agreement with the results of Ishiguro (2008), Fulle et al. (2010), and Agarwal et al. (2010), whose dust production rates range from $180-1000 \mathrm{~kg} \mathrm{~s}^{-1}$. We discuss below why we consider an $\alpha \gtrsim-4$ to be a better representation of the available data.

\subsection{Trail profiles}

We compared trail profiles along and perpendicular to the projected orbit of the comet. We compared the modeled and observed trails for the cases given by the 2004 Spitzer observation reported by Kelley et al. (2008) and by the second Spitzer observation (2006) described in Agarwal et al. (2010). The modeled profiles were scaled to the maximum of the observed profiles to facilitate a comparison of the profile shapes. Figures 4 and 5 


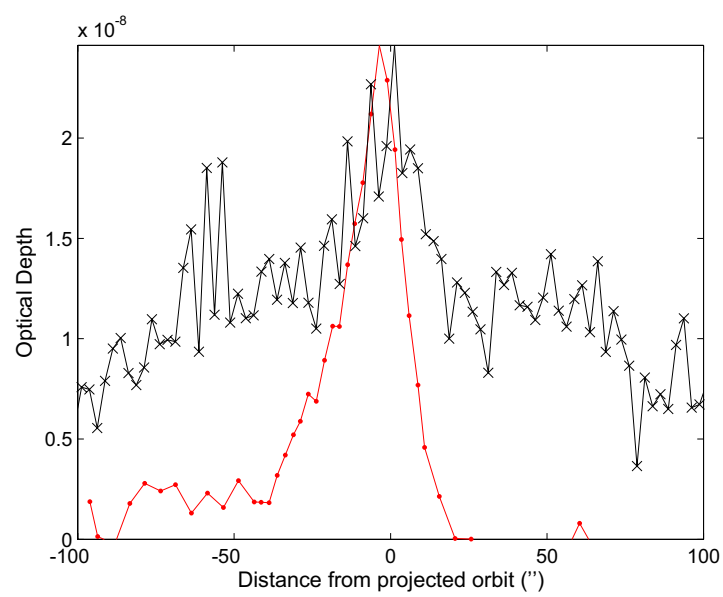

(a)

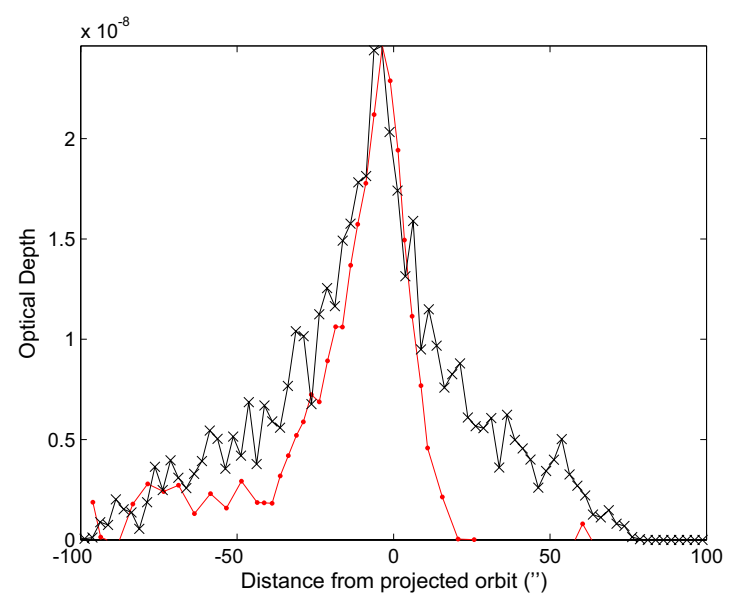

(c)

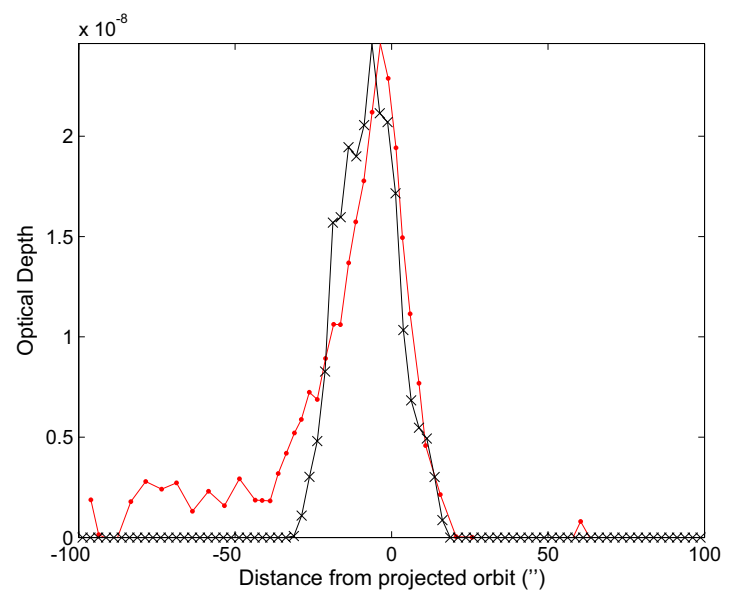

(e)

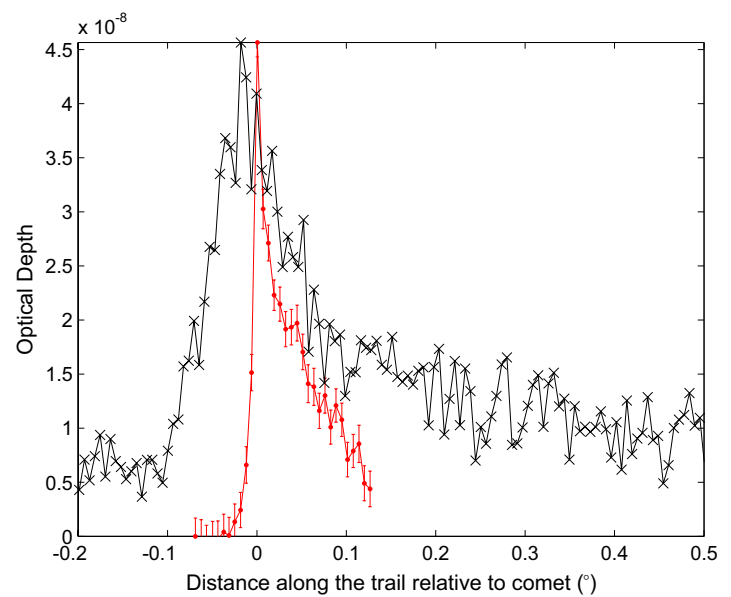

(b)

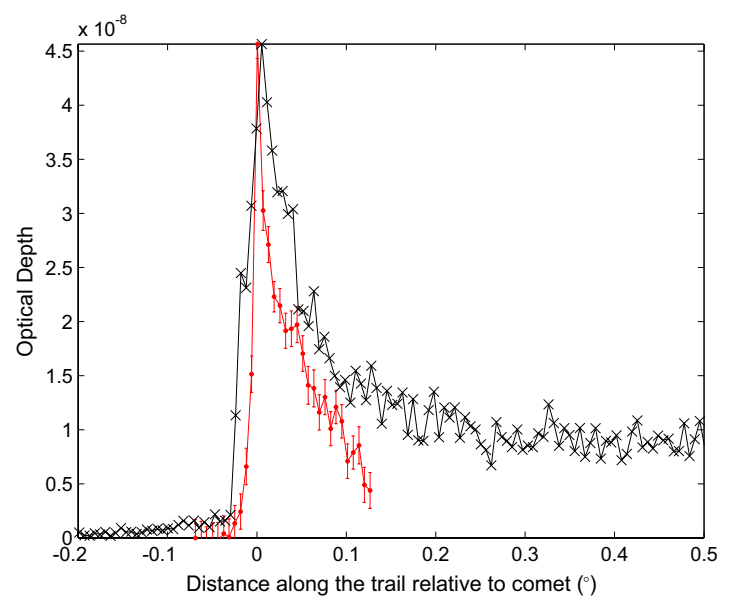

(d)

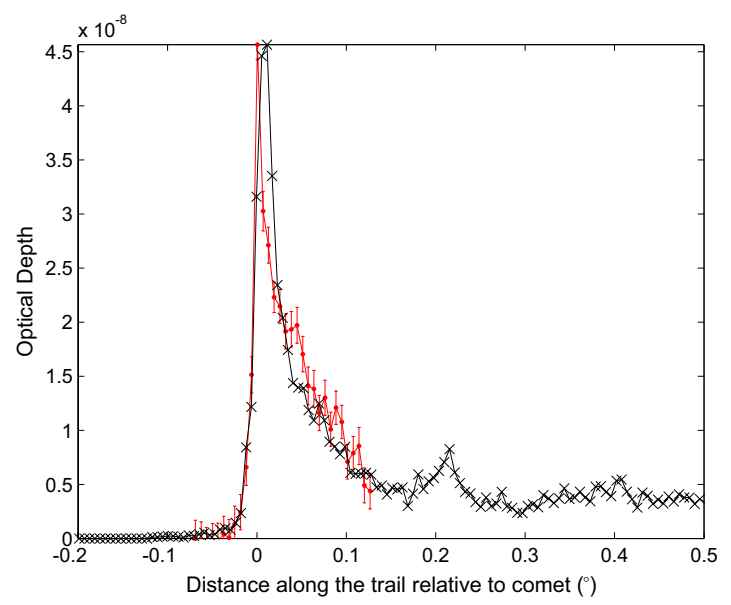

(f)

Fig. 4. Comparison of modeled (black) and observed (red) optical depth cross-sections across (a), c), e)) and along (b), d), f)) the trail for the 2004 Spitzer observation of the 67P trail described in Kelley et al. (2008) for three velocity models: (a), b)) Crifo \& Rodionov (1997); (c), d)) Kelley et al. (2008); (e), f)) Agarwal et al. (2010). For (b), d), f)) the positive direction is the trailing direction of the comet movement. For (a), c), e)) the cut is perpendicular to the projected comet orbit, with positive values in the direction of positive declination. A size distribution with $\alpha=-3.7$ is used.

provide profiles for three velocity models for the 2004 and 2006 observations. In most cases, the velocity model of Agarwal et al. (2010) provides the best match to the observations. The profiles perpendicular to the trail are most diagnostic and demonstrate that a low velocity is required to fit the data. A second indicator for the velocity model is the section of the profile along the trail that is in front of the comet.

While the profiles perpendicular to the trail are largely only dependent on the velocity, the profiles along the trail are dependent on a combination of the velocity and the mass distribution. 
R. H. Soja et al.: Characteristics of the dust trail of 67P/Churyumov-Gerasimenko: an application of the IMEX model
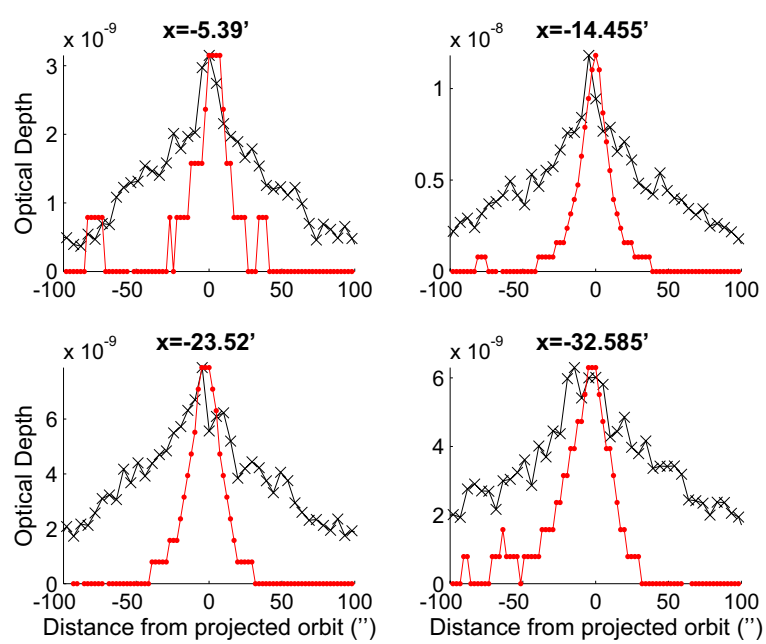

(a)

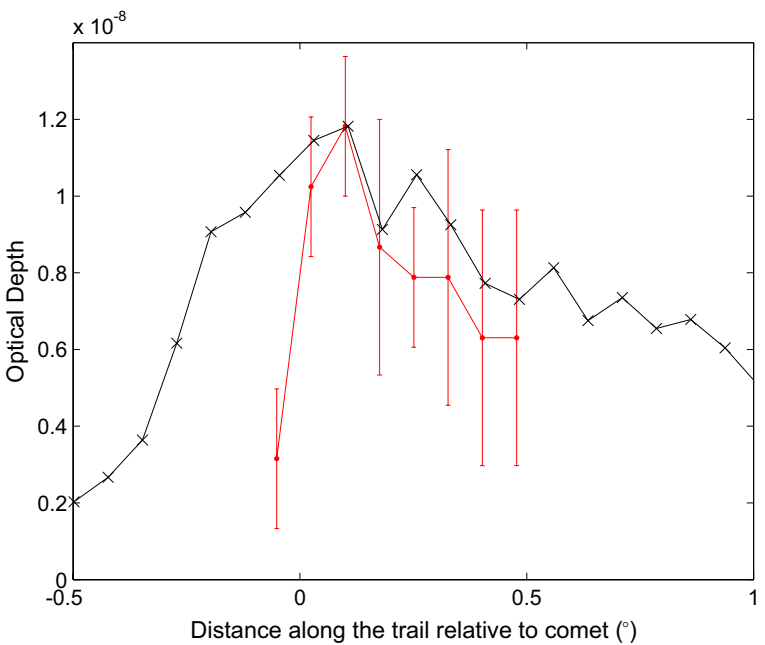

(b)
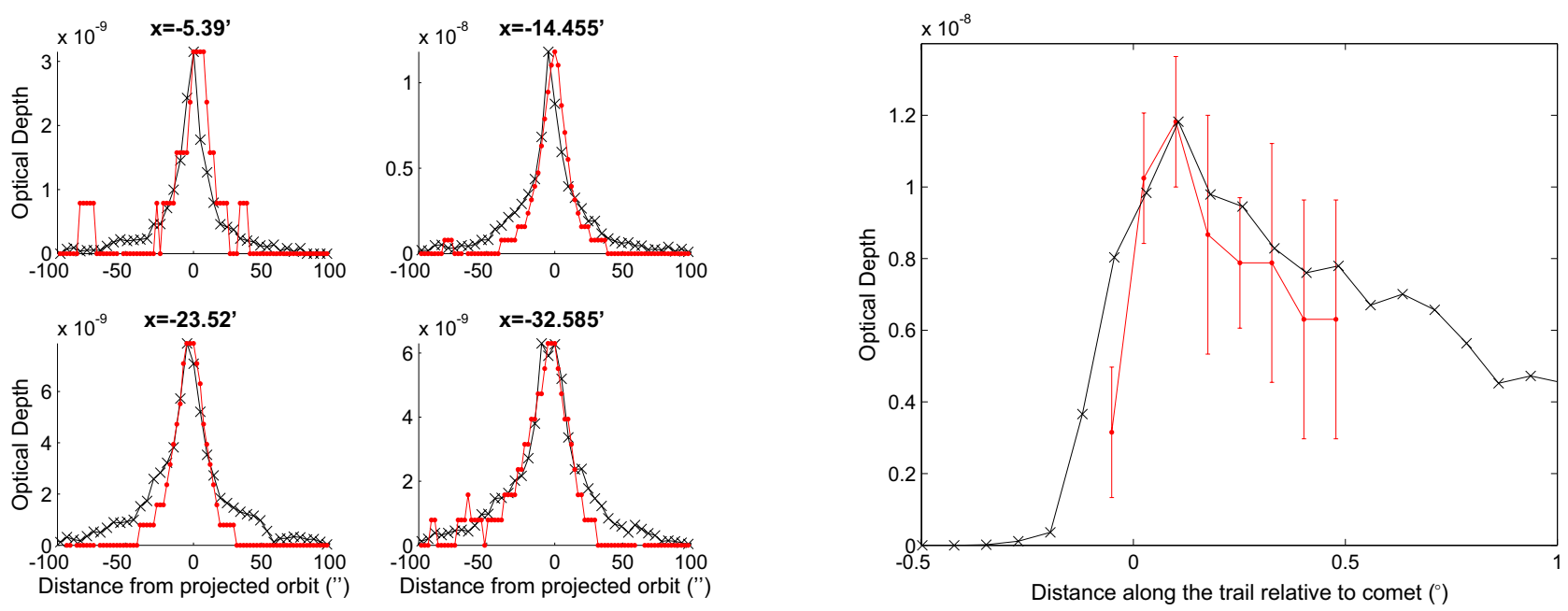

(c)
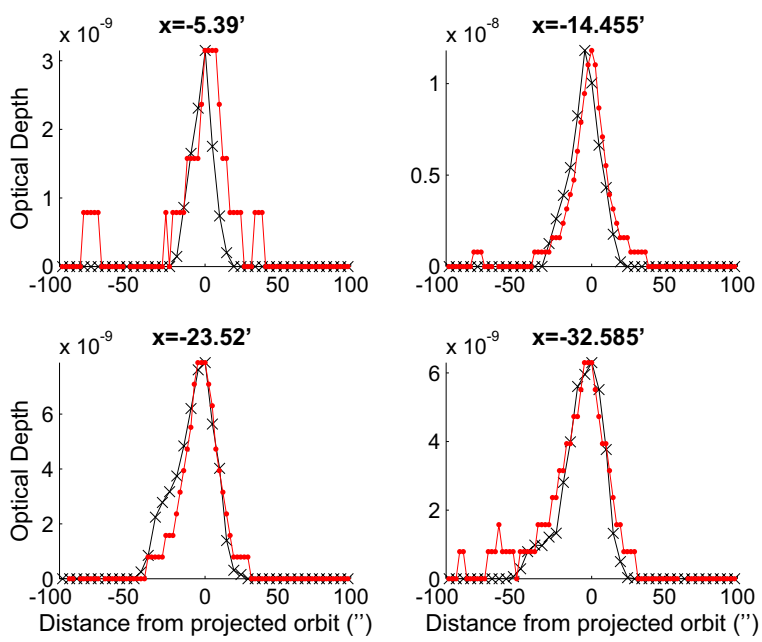

(e)

(d)

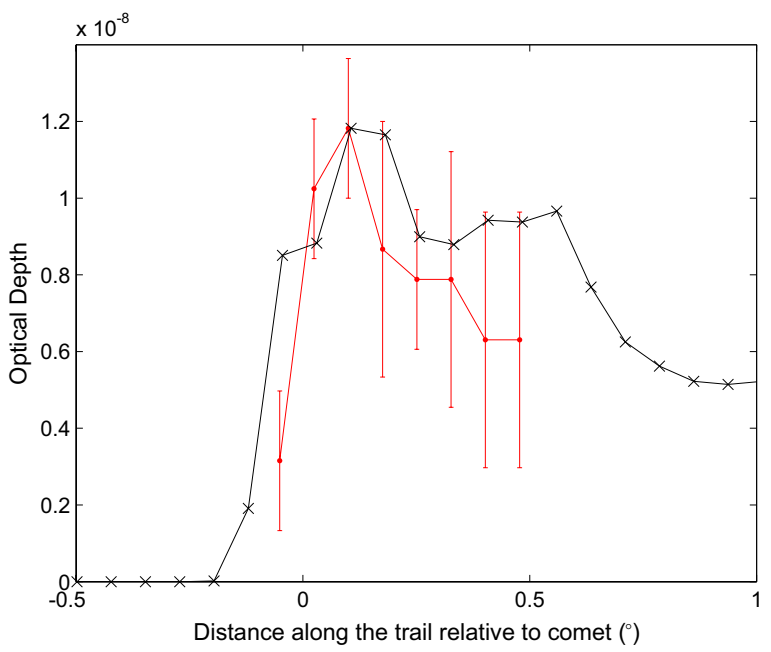

(f)

Fig. 5. Comparison of modeled (black) and observed (red) optical depth cross-sections across (a), c), e)) and along (b), d), f)) the trail for the 2006 Spitzer observation of the 67P trail described in Agarwal et al. (2010) for three velocity models (a), b) Crifo \& Rodionov 1997; c), d) Kelley et al. 2008; e), f) Agarwal et al. 2010). Panels in (a), c), e)) refer to the angular distance ( $x$, as used by Agarwal et al. 2010) from the comet along the trail at which the cross-section cut is taken. For (b), d), f)) the positive direction is the trailing direction of the comet movement. For (a), c), e)) the cut is perpendicular to the projected comet orbit, with positive values in the direction of positive declination. The Spitzer observations discussed in Agarwal et al. (2010) are limited to within $\sim 30^{\prime}$ of the comet. 


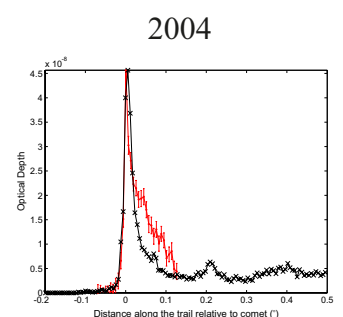

(a) $\alpha=-2.9$

2006

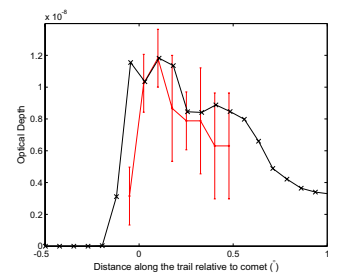

(e) $\alpha=-2.9$
2004

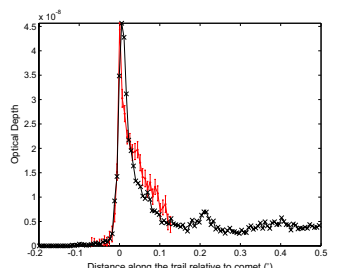

(b) $\alpha=-3.5$

2006

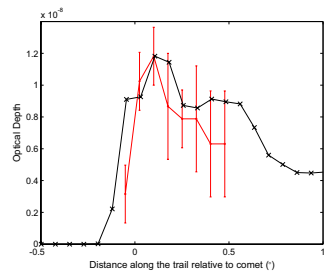

(f) $\alpha=-3.5$
2004

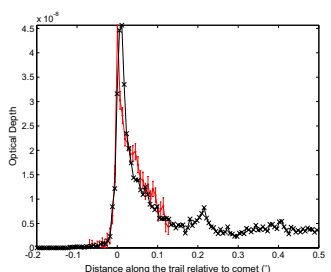

(c) $\alpha=-3.7$

2006

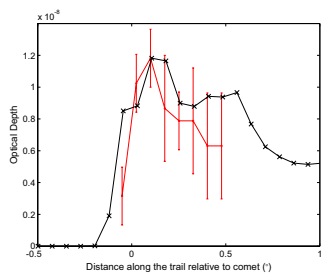

(g) $\alpha=-3.7$

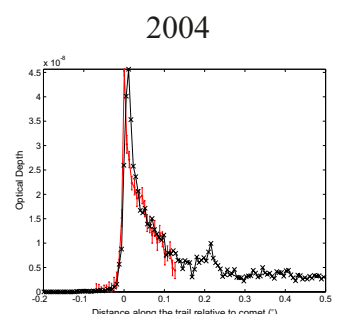

(d) $\alpha=-4.1$

2006

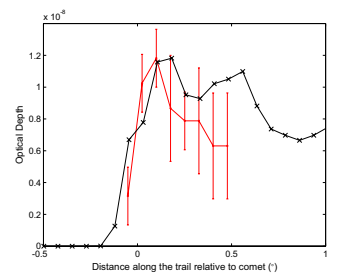

(h) $\alpha=-4.1$

Fig. 6. Comparison of modeled (black) and observed (red) optical depth cross-sections along the trail for the 2004 and 2006 Spitzer observation of the 67P trail described in Kelley et al. (2008) and Agarwal et al. (2010): using the velocity model of Agarwal et al. (2010) for differential size distribution indices $\alpha=-2.9,-3.5,-3.7$, and -4.1 . The positive direction is the trailing direction of the comet movement.

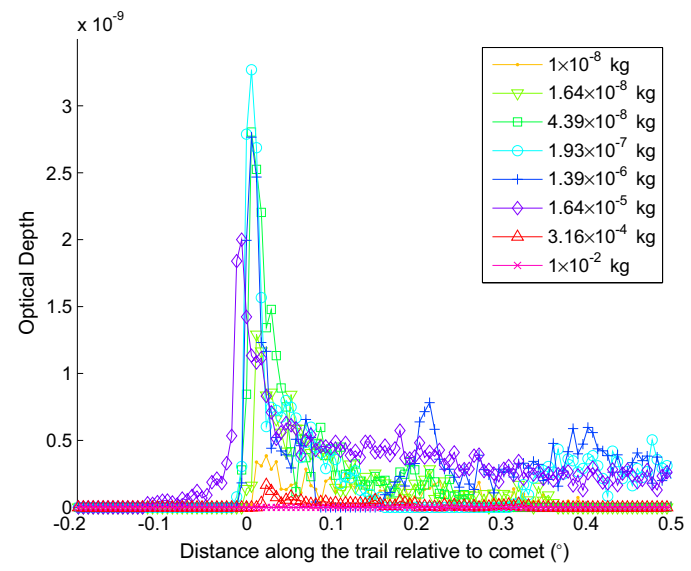

(a)

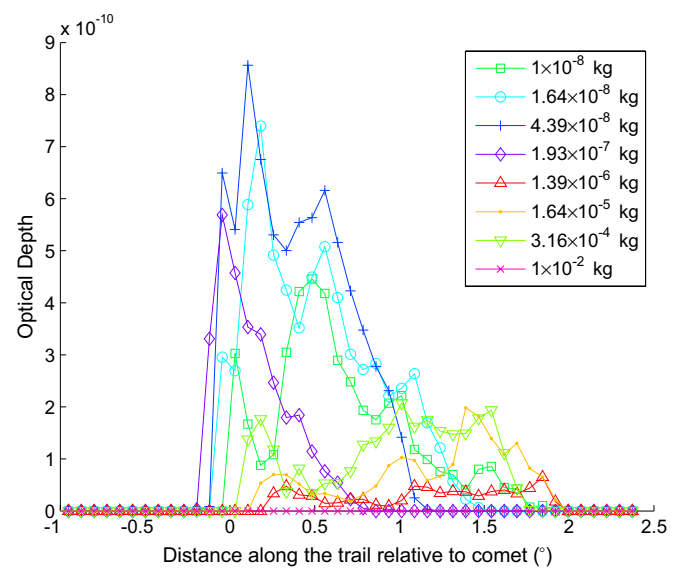

(c)

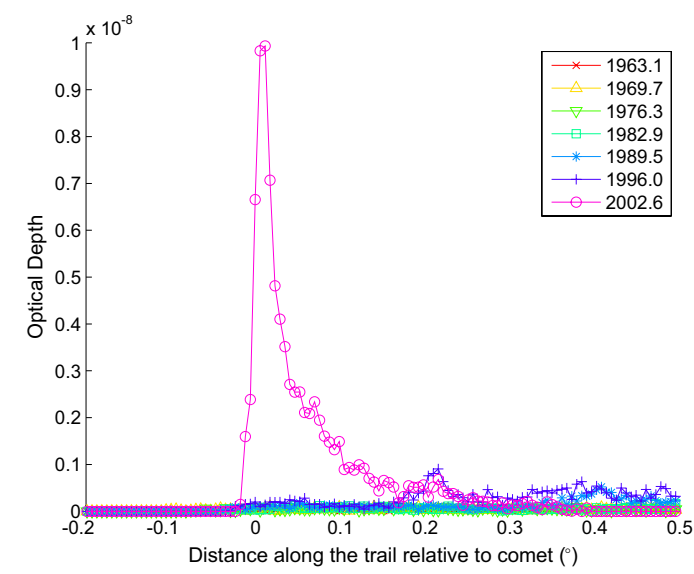

(b)

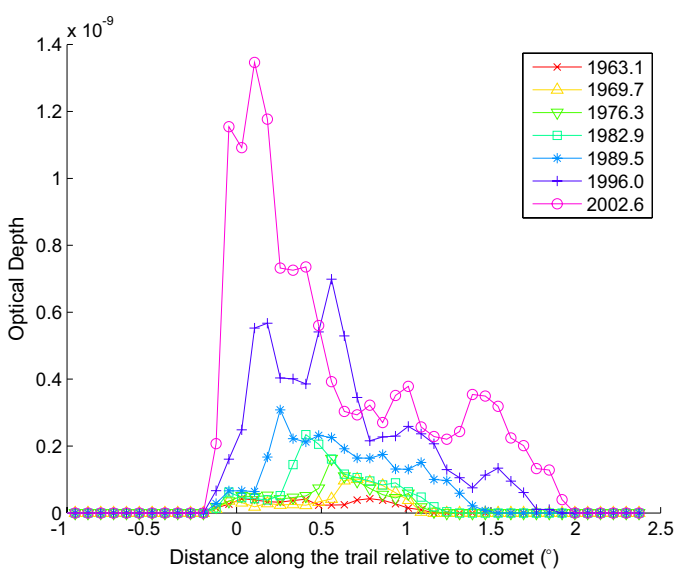

(d)

Fig. 7. Modeled optical depth cross-sections along the trail for the 2004 (a), b)); and 2006 (c), d)) Spitzer observation of the 67P trail (Kelley et al. 2008; Agarwal et al. 2010) using the velocity model of Agarwal et al. (2010). (a), c)) show the contribution of particles of different masses to the total optical depth; (b), d)) show the contribution of particles created during different comet apparitions to the total optical depth. The positive direction is the trailing direction of the comet movement. 
R. H. Soja et al.: Characteristics of the dust trail of 67P/Churyumov-Gerasimenko: an application of the IMEX model

1986

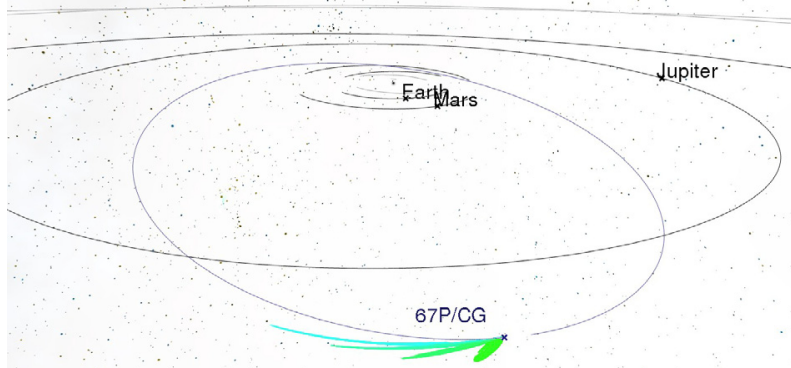

2021

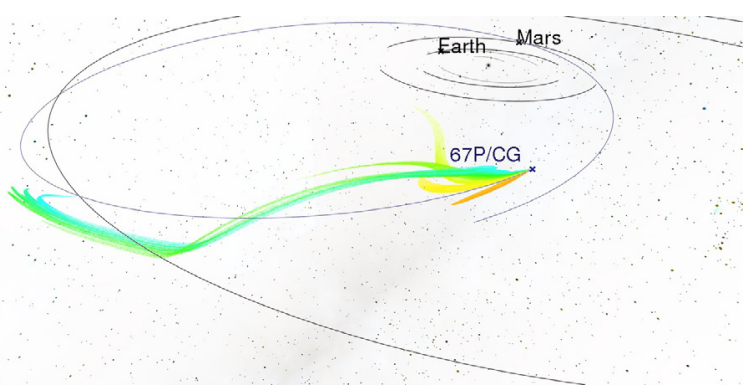

2069

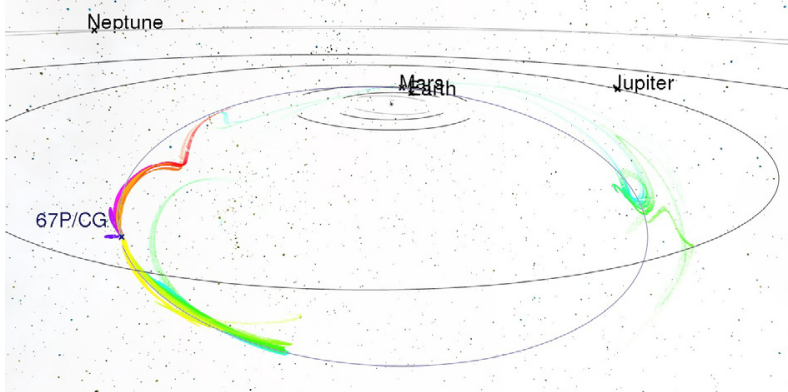

2096

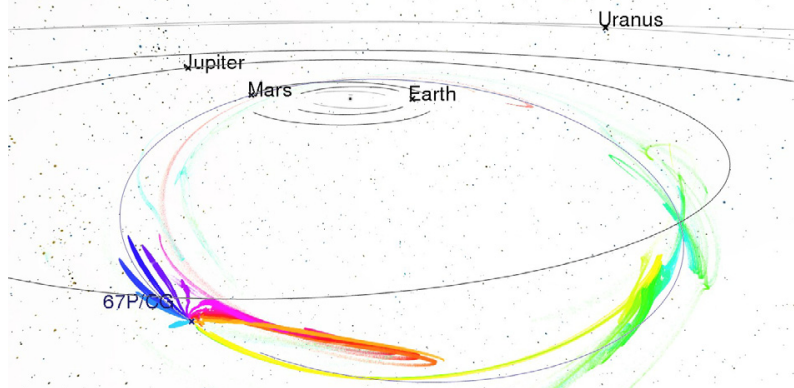

2011

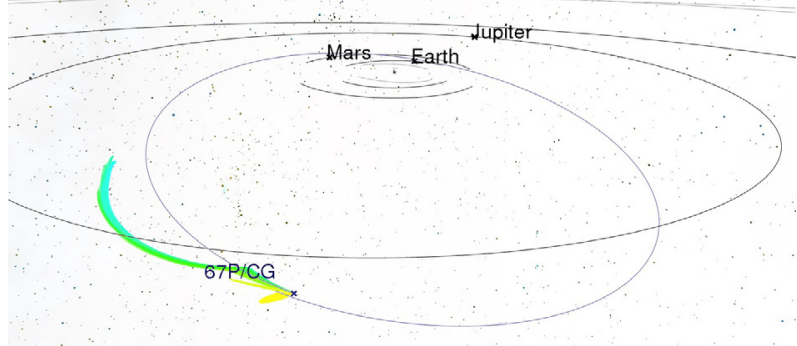

2040

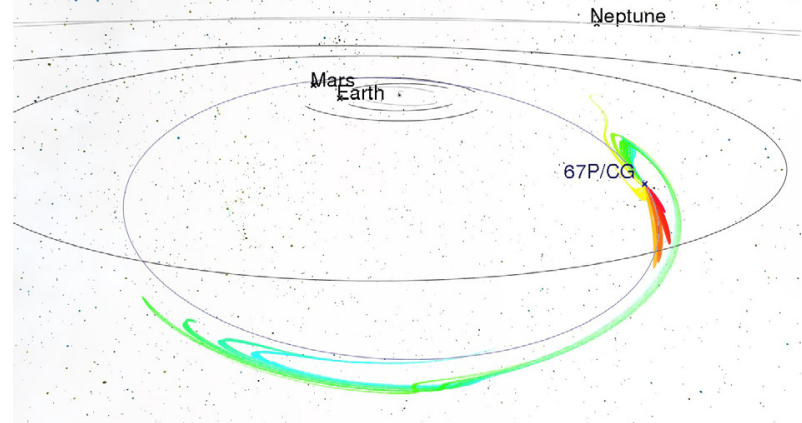

2091

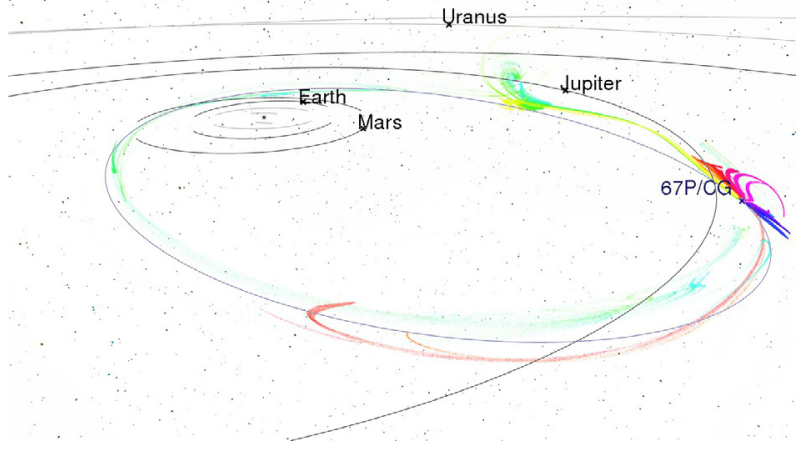

2099

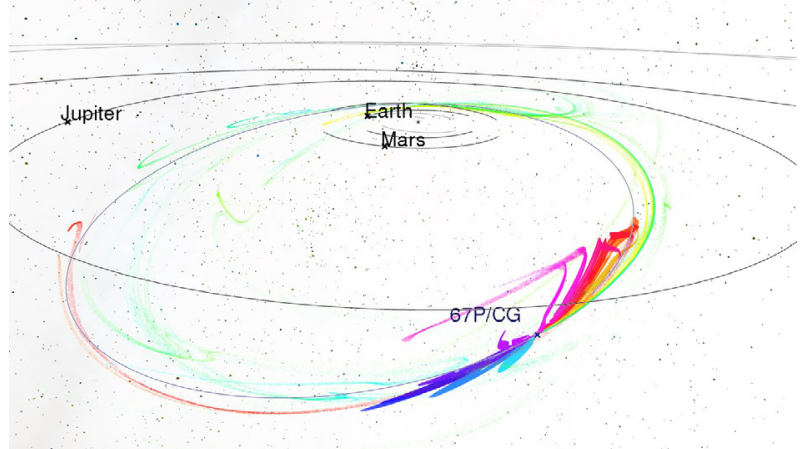

Fig. 8. Development of the meteoroid streams of comet 67P/Churyumov-Gerasimenko between 1986 and 2099. Colors are particles released during different apparitions (green particles are oldest, blue particles are youngest). We also show the orbits of eight planets and the comet. We see evidence of close encounters with Jupiter that disperse particles and warp the stream. In 2069 the tail end of the red streams behind the comet display gaps that are indicative of Mars' near passage through the stream in January 2062 (Vaubaillon \& Colas 2005). These are stills from a video of the whole stream from 1959 to 2100 that can be viewed at https://vimeo.com/128363607; and in the online edition of the journal. 
Figure 6 demonstrates how the 2004 and 2006 profiles vary for $\alpha=-2.9--4$.1. Including the error bars (which represent $1 \sigma$ of the background variation), the 2004 profile is matched best by $\alpha=-3.7--4.1$, and the 2006 profile by $\alpha=-2.9--3.7$. An intermediate $\alpha=-3.7$ is appropriate for both datasets.

\subsection{Profiles as a function of mass and release date}

Here we examine the contributions to the total profiles along the projected orbit by (a) particles of different masses; and (b) particles released during different comet apparitions (Fig. 7).

Two main features are apparent in mass profiles. The brightness is dominated by particles $\sim 1.93 \times 10^{-7}-1.64 \times 10^{-5} \mathrm{~kg}$ for the 2004 observation, and $\sim 1.39 \times 10^{-6}-1.64 \times 10^{-5} \mathrm{~kg}$ for the 2006 observation: particles $\sim 0.5-1.6 \mathrm{~mm}$ in radius. Furthermore, the 2006 profile shows evidence that the smallest grains $\left(<10^{-7} \mathrm{~kg}\right.$ or $\left.\$ 300 \mu \mathrm{m}\right)$ are already falling behind the comet and leaving the trails. Most of these grains are outside of the observed region $\left(<0.5^{\circ}\right)$. This behavior is not seen to the same extent in the 2004 profile because the most recently released particles have not yet had time to disperse behind the comet.

Both the 2004 and 2006 profiles are dominated by the most recently emitted particles: those from the 2002.6 apparition. This behavior is more pronounced for 2004 because this date is closer to the release date of these particles and no significant dispersion of their orbits has occurred yet. For the 2006 profile, the optical depth contribution of the particles released during the second to last apparition (1996.0) is $\sim 40 \%$ of the contribution from the 2002.6 apparition. The contribution of particles from the 1989.5 apparition is $15 \%$ of the 2002.6 apparition. This behavior is also demonstrated by the flatter profile of the 2006 observation.

\section{Discussion}

Our modeling of the trail of comet 67P/ChuryumovGerasimenko demonstrates that the IMEX model can be used to fit observations of cometary trails. Our approach does not test all possible sets of parameters and is not intended to provide new information on the trail. Instead, it demonstrates that we are able to match main features of the comet trail with our simple model.

Our model provides the best fit with a very low velocity model and a moderate size distribution index of $\alpha=-3.7$. This compares to the $\alpha=-3.5$ found by Kelley et al. (2008) and Ishiguro (2008), $\alpha=-4.1$ found by Agarwal et al. (2010). Fulle et al. (2004) found a size distribution that changes from $\alpha=-3.5$ before perihelion to -4.4 after perihelion. There is observational evidence that the dust production parameters do vary pre- and post-perihelion. We note that these models do not use a broken size distribution with a "knee" dividing the behavior of large and small grains, as in the IMEX model. However, a two-part mass distribution with $\alpha=-3$ for smaller particles and $\alpha=-4$ for larger particles is used in the dust environment model employed for GIADA mission planning (Fulle et al. 2010).

The best-fit velocity model (that of Agarwal et al. 2010) is about a factor of two lower at perihelion than the models of Kelley et al. (2008) and Ishiguro (2008). While Fulle et al. (2004) found a difference between pre- and post-perihelion ejection speeds, Fulle et al. (2010) found that a perihelion-symmetric velocity is consistent with available data. Additionally, the heliocentric distance dependence of the model of Agarwal et al. (2010) is much steeper than all other models. While the $r_{\mathrm{h}}^{-1 / 2}$ dependence of most models represents the variation in solar insolation with heliocentric distance, the model of Agarwal et al. (2010) uses the stronger heliocentric distance dependence of the comet's water production to define a $r_{\mathrm{h}}^{-3}$ dependence. Fulle et al. (2010) also found that the steep heliocentric dependence of the velocity model of Agarwal et al. (2010) is inconsistent with available trail and coma data.

Our dust production is also managed very differently compared to previous models. Other work defined or fitted a heliocentric distance dependence for the dust production rate: Ishiguro (2008) found the data to be best fit by $r_{\mathrm{h}}^{-3}$, Kelley et al. (2008) used $r_{\mathrm{h}}^{-5.8}$, and Agarwal et al. (2010) assumed $r_{\mathrm{h}}^{-8}$. Here our dust production is defined by the total magnitude (11.4) and the magnitude slope parameter (11) for the comet, as given by the JPL SBDB. The resulting dependence for the dust production is $r_{\mathrm{h}}^{-2.7}$, which is slightly lower than other models. Additionally, Fulle et al. (2010) found that the dust mass-loss rate is asymmetric around perihelion as a result of the change in the mass distribution. Our model assumes the same behavior before and after perihelion.

The IMEX model is designed to provide only an approximation to each trail: it is not intended to be highly accurate in all cases. Indeed, this is currently not possible. We know that parameters such as the ejection velocity, mass distribution, and dust production rate - all potentially as a function of time relative to perihelion and apparition - are likely to vary for each comet. Currently, individual comets are found to require very different ejection velocity models (Ryabova 2013). We therefore do not expect high-precision accuracy for the general model. However, this test case demonstrates that the model can be efficiently refined for streams of particular interest to encompass higher quality information on individual comets.

This study therefore demonstrates that the IMEX model is successful in fitting observational data for cometary streams when the basic cometary parameters - mass distribution and ejection velocity - are already constrained.

If no specific comet is identified, such as in the case of a future spacecraft mission, the full IMEX database can be used to determine streams in the vicinity of point in space or time. When the comet of interest is known, IMEX can be used in conjunction with observational data, such as infrared trail images or meteor shower data, to determine refined parameters for the comet. The existing IMEX database provides a first-step model for the stream of interest. When a good match is not achieved, the integrator and other tools can be used to recalculate the stream using different cometary parameters.

\section{Future directions for the model}

The model will provide an unprecedented data set of meteoroid streams in the inner solar system. This has benefits not only for impact hazard assessment for spacecraft hardware. It can also be used to study the development of typical meteoroid streams with time (as demonstrated in Fig. 8 for 67P/ChuryumovGerasimenko); to study "meteor storms" occurring at any spacecraft, planet or other body in the inner solar system, creating perhaps a meteor shower there on this date; and to understand the development of these trails into the background. The observed gaps in the 67P/Churyumov-Gerasimenko trail in Fig. 8 are caused by the passage of Mars within 4 Mars radii of the 67P/Churyumov-Gerasimenko trail in January 2062, which may create such a meteor event at Mars. Work is ongoing to examine the performance of the IMEX model in matching observations 
of recent meteor storm events at Earth. In particular, the model may be useful for operators of scientific instruments on spacecraft, who may need to be aware of the danger of such streams, of the affect of dust on measurements, or of dust stream observation opportunities.

Acknowledgements. This work was funded under ESA contract 4000106316/12/ NL/MV - IMEX, and supported by Constellation, a young academics group supported by the German Society for Aeronautics and Astronautics DGLR in Stuttgart, Rechenkraft.net e.V. and Selfnet e.V. We thank all individual Constellation users for their computing time.

\section{References}

Agarwal, J., Müller, M., \& Grün, E. 2007, Space Sci. Rev., 128, 79 Agarwal, J., Müller, M., Reach, W. T., et al. 2010, Icarus, 207, 992

A'Hearn, M. F., Schleicher, D. G., Millis, R. L., Feldman, P. D., \& Thompson, D. T. 1984, ApJ, 89, 579

A'Hearn, M. F., Millis, R. L., Schleicher, D. G., Osip, D. J., \& Birch, P. V. 1995, Icarus, 118,223

Crifo, J. F., \& Rodionov, A. V. 1997, Icarus, 127, 319

Crovisier, J., Colom, P., Gérard, E., Bockelée-Morvan, D., \& Bourgois, G. 2002, A\&A, 393, 1053

Dikarev, V., Grün, E., Baggaley, J., et al. 2005, Adv. Space Res., 35, 1282

Divine, N. 1981, in The Comet Halley. Dust and Gas Environment, eds. B. Battrick, \& E. Swallow, ESA SP, 174, 47

Divine, N., \& Newburn, Jr., R. L. 1987, A\&A, 187, 867

Dormand, J. R., \& Prince, P. J. 1978, Celest. Mech., 18, 223

Ertel, S., Wolf, S., \& Rodmann, J. 2012, A\&A, 544, A61

Feldman, P., A'Hearn, M., \& Festou, M. 2004, in The New Rosetta Targets. Observations, Simulations and Instrument Performances, eds. L. Colangeli, E. Mazzotta Epifani, \& P. Palumbo, Astrophys. Space Sci. Lib., 311, 47
Fulle, M., Barbieri, C., Cremonese, G., et al. 2004, A\&A, 422, 357 Fulle, M., Colangeli, L., Agarwal, J., et al. 2010, A\&A, 522, A63

Gundlach, B., Blum, J., Keller, H. U., \& Skorov, Y. V. 2015, A\&A, 583, A12

Gustafson, B. A. S. 1994, Ann. Rev. Earth Planet. Sci., 22, 553

Ishiguro, M. 2008, Icarus, 193, 96

Jorda, L., Crovisier, J., \& Green, D. W. E. 2008, LPI Contributions, 1405, 8046 Kelley, M. S., Reach, W. T., \& Lien, D. J. 2008, Icarus, 193, 572

Kidger, M. R. 2004, A\&A, 420, 389

Kresak, L. 1993, A\&A, 279, 646

Langland-Shula, L. E., \& Smith, G. H. 2011, Icarus, 213, 280

Mäkinen, J. T. T. 2004, in The New Rosetta Targets, Observations, Simulations and Instrument Performances, eds. L. Colangeli, E. Mazzotta Epifani, \& P. Palumbo, Astrophys. Space Sci. Lib., 311, 61

Molina, A. 2010, Rev. Mex. Astron. Astrof., 46, 323

Reach, W. T., Kelley, M. S., \& Sykes, M. V. 2007, Icarus, 191, 298

Rodmann, J. 2006, Ph.D. Thesis, Combined Faculties for the Natural Sciences and for Mathematics of the University of Heidelberg, Germany

Rotundi, A., Sierks, H., Della Corte, V., et al. 2015, Science, 347, 3905

Ryabova, G. O. 2013, Sol. System Res., 47, 219

Sanzovo, G. C., Singh, P. D., \& Huebner, W. F. 1996, A\&AS, 120, 301

Sanzovo, G. C., Sanzovo, D. T., \& de Almeida, A. A. 2010, in IAU Symp. 263, eds. J. A. Fernandez, D. Lazzaro, D. Prialnik, \& R. Schulz, 272

Schleicher, D. G. 2006, Icarus, 181, 442

Schulz, R., Hilchenbach, M., Langevin, Y., et al. 2015, Nature, 518, 216

Singh, P. D., de Almeida, A. A., \& Huebner, W. F. 1992, AJ, 104, 848

Snodgrass, C., Tubiana, C., Bramich, D. M., et al. 2013, A\&A, 557, A33

Sykes, M. V., \& Walker, R. G. 1992, Icarus, 95, 180

Sykes, M. V., Lebofsky, L. A., Hunten, D. M., \& Low, F. 1986, Science, 232 1115

Vaubaillon, J., \& Colas, F. 2005, A\&A, 431, 1139

Vaubaillon, J. J., \& Reach, W. T. 2010, AJ, 139, 1491

Weiler, M., Rauer, H., \& Helbert, J. 2004, A\&A, 414, 749

Yeomans, D. K., Chodas, P. W., Sitarski, G., Szutowicz, S., \& Królikowska, M. 2004, in Cometary orbit determination and nongravitational forces, ed. G. W. Kronk, 137 